\title{
La noción de pueblo en el discurso populista
}

\section{MARLA FERNANDA MADRIZ}

UNIVERSIDAD CENTRAL DE VENEZUELA

RESUMEN. Esce articulo resume los resultados de una investigación cuyo ubierivo fue escablecer los rasgos semánticos relevantes de la caregoría de pueblo. ral cual ésra ha venido siendo construida por los principalés lidéres pulícicos venezulanus del siglo XX. El escudio se llevó a cabo desde el marco rransdisciplinas del análisis crírico del discurso y ruvo cumu corpus lus discursos del señor Rómulo Berancourt -lundador del parcido Acción Democrárica- y del actual Presidente de la República, reniente coronel Hugo Chávez Frlas. El análisis permició concluir que el pueblo se ha asociado en el discurso populista venezolano a unidades de significación no sólo diversas sino abiertamente contradictorias que lu idencifican como: 1) el sujeco histórico de su propia liberación, en cuyo caso se inscicuye como "cíclope"; 2) el objeto del maluraro, la desidia y la manipulación del poder, en cuyo caso se insticuye como "mártir" $y$; 3) el descinarario de la dádiva del gobierno, en cuyo caso se insticuye como "ererno beneficiario".

palabras Clave: pueblo, discurso populista, Betancourt, Chávez, Venezuelo.

RESUMO. Este arriculo resume os resultados de una pesquisa, eujo objerivo foi esrabelecer os rraços semáncicos relevantes da carcgoria do povo, ıal qual esra foi consiruida pelos principais líderes políricos venezuelanos, do século XX. $O$ escudo se levo a cabo desde o marco transdisciplinar da análise crírica do discursn e reve como corpus os discursos poferido pelo Sr. Rómulo Betancourt -fundador do parcido Ação Democrárica-e do atual Presidente da República, tenente coronel Hugo Chávez Frías. A análise permiriu concluir que o povo foi associado, no discurso populista venezuclano, a unidades de significa ção nãu só diversas senàn aber tamente contraditórias a ponto de identificá-lo como: 1)o sujeito histórico da súa própria libercação, em tal caso se institui como "ciclope"; 2) o objero do malıraro. a disidia e d manipulação do poder, em ral casn se inscícui como "márrir" e; 3) u descinátarios da dádiva do governo, em ral caso se insrioui como "eterno beneficiário".

pat.avras chave: povo, discuroo populsta, Betancourt, Chavez, Venezuela. 
ABSTRACT. This arricle summarizes the resules of an investigation whose ubjecrive was ro identify the most relevant semanric features of the caregory "people", as this has been constructed by the main political leaders of the XXth century in Venezuela. The study was carried out within the eransdisciplinary framework of critical discourse analysis. The corpus consisted of speeches pronounced by Rómulo Berancourt -the founder of Acción Democrática (Democratic Action) party-and by the present president of the Republic, Hugu Chávez Frías. The analysis allows us to cunclude that "the peuple". in populist discuurse, is assuciated, with diverse and openly contradictory units of meaning that identify it as: 1) the historic subject of its uwn liberation, in which case it is consrituted as a "cyclop"; 2) the object of maltreatment, neglect and manipulation of power, in which case ir becomes a martyr, and 3) the rarger of the government's "gifts", in which case ir becomes the eternal beneficiary.

KeYwords: People, populisz discourse, Betancourt, Chávez. Venezuela.

\section{Introducción}

El líder se sacude los sudores que le empapan la frence y observa, satisfecho, la mulrirud de ojos que le observan. La plaza hierve por la severidad del clima y por el éxtasis tebril de los que escuchan el discurso. El Irder ha elegido las palabras que permiten remplar ambas temperaruras: la de la atmósfera y la de la emoción. Previendo que el calor le espante los adeptos, el líder ha pedido "que me escuchen; que soporten cinco minuros más este agresivo sol [...] de mediodía", arguyendo que "me queda el consuelo de que yo rambién ló esroy soportando" (B-47).

Receloso de que el cansancio le mengüe los ardores a los prosélitos, el líder ha ensalzado la muchedumbre $y$ ha adverrido que:

Quien lea la prensa [... encuntrará por alli afirmaciones rotundas en el senrido de que en Venezuela no ha habido una Revolución [...] Pero el supermo juez en una democracia, que es el pueblo, está diciendo aquí con su presencia [...] que las mayorlas populares de nuestra patria conflan hoy más que nunca en [...] quienes ejercen, por soberana decisión suya, [el poder] . (B-71)

Y finalmente, sabiendo de antemano lo que "las mayorias populares de nuestra parria" desean escuchar, el líder ha dado garantías de que en el país gobierna:

un equipo de hombres civiles y militares [...] a quienes no les vacilará el pulso ni les remblará la mano para llevar a la Revolución hasıa sus objerivos: devolver al pueblo su soberanía, devolver al pueblo su derecho al pan y a la cultura sin regateos, devolver al pueblo venezolano su derecho a disfrutar de justicia social. (B-22)

No. El líder a quien ciro no es Hugo Chávez Frías, en los albores del siglo XXI, exaltando los dones de la "revolución bolivariana". Es Rómulo 
Betancourt, en 1947, dilapidando loas por la "revolución de octubre". Y, el aire de semejanza que acerca sus discursos, refiere a lo que comúnmente llamamos "populismo".

El fenómeno remice a un modo parricular de hacer polírica, de insrituir, organizar y regular las relaciones de poder entre gubernantes y gobernados. Según algunos estudiosos del tema cuyos enloques hallamus en especial sugerentes (Rey, 1976́, 1980̄: Bricto García 1988. 1989), uno de los arriburos disrincivos del modelo populisra es que éste funda y peserva su legirimidad en dos ripos de prácricas que aseguran la circulación social de dos tipos distintos y a la vez articulados de bienes.

La primera de estas prácricas es de carácier ecunómico y alude a la distribución de los recursos del Esiadu que, en la forma de servicios y bienes mareriales, los dirigentes reparten entre los dirigidos. Señala al respecro Juan Carlos Rey:

Tál repartu no riene que hacerse en parres necesariarnente iguales [...] pür el conirario, lo típico es que los sectores más marginados y desorganizados parricipen en proporción cunsiderablemenre inferior que los más organizados y privilegiadus de manera que, a la larga. el resultado general de las polficas redistriburivas es el aumento de la brecha entre ambos seciores (Rey, 1980: 162)

La posible conversión de tal brecha en abismo y, de alli, en anragónica lucha polírico-social, explica por qué el modelo populista requiere de recursos estacales excedentarios, si es que se precende que éste logre implanrarse con éxico. De orro modo, la imposibilidad de repartir entre los dirigidos los anhelados bienes, terminará por alejarlos de sus gobernanres, produciéndose lo que Antonio Gramsci llamó una crisis de begemonia que. a la postre, renderá a resolverse con la defenestración del grupo en el poder.

Podrá argumentarse que lo dicho no es un rasgo exclusivo del modelo populisra, visio que roda elire dirigenre funge de adminisrradora de los recursos estacales e invierte en obras que a la larga redundan en algún benelicio para la población en general

Podrá argumentarse tal cosa y será cierro. Nuestra hipótesis de trabajo es precisamente que, en el populismo, la rurinaria administración de los recursos del Esıado por parte del equipo gobernance adquiere un cariz diferente y cumple una función discinca a aquéla cumplida por esta práctica económica bajo otros modelos de ejercicio del poder. Y, lo que hace posible que cal meramorfosis se consume, es precisamence la apelación regular y sistemárica por parte de los gobernanres al segundo ripu de prácrica social a la que aludiamos cres párrafos arriba, a saberi, a la comunicación colecriva, a las prácricas discursivas de carácrer público

En efecto, es gracias a estas úlrimas que los individuos generan. internalizan e incercambian un tipo singular de bienes Cascoriadis 
1988, 1989. 1998) lus ha llamado ignificaciones sociales imaginariasque nu son otra cusa más que el conjunto de significados que integran los diversos modelos de incerpretación con base en los cuales los miembros de una sociedad se explican el mundo, la vida. el lugar que cada quien ocupa en esa vida. En las sociedades contemporáneas, el circuito hegemónico -aunque no exclusivo- a través del cual los actores sociales producen, incercambian y eventualmente instituyen estas significaciones sociales o modelos de interprecación es el del sisrema de medios masivos

Para el caso que nus atañe -el de la pulfrica- el discurso populista que circula a rravés de escos medios instituye un curioso modelo de interpreración de las relaciones de poder merced al cual, el grupo o el líder gobernanıe devienen magnánimos benefacrores de lo que llamaré junto a Britro García (1988) la "dádiva", al tiempo que los gobernados -adicros a esia úlrima- devienen por su intermedio en fervorosos vasallos de una lealtad erigida ora sobre el oportunismo, ora sobre la gratitud.

Nos explicamos. En aquellus casos en los que el poder no se ejerce con arreglo al modelo populista, ante los ojos y la conciencia de los ciudadanos quedan pocas dudas sobre el origen de los recursos que se distribuyen, las obligaciones de aquellos que los distribuyen y los derechos de quienes resultan sus destinatarios. El ciudadano común sabe que la élite en el poder administra -con mayor o menor equidad- una riqueza que es de la República; está más o menos persuadido de que el grupo dirigente cumple una función pública para la cual ha sido electo por sus representados $y$, por consiguiente, es evaluado por éstos con base en los logros o errores de su gesción. En escos casos, el vínculo que une los gobernados a sus gobernanres es la sarisfacción y conformidad -más o menos conscience y justificada- de los primeros, con los resultados concretos obtenidos por los segundos.

En el caso del populismo, por el contrario, el lider gobernance deviene-gracias a las prácricas discursivas masivamente comunicadas- en una suerte de mecenas que reparte "obsequios" entre la población. Léase bien: Es el uso sistemárico de ciertas entraregias reróricas por parte del líder populisra lo que en lo fundamenral hace posible que ésce no sea socialmente aprehendido como un funcionario público que adminiscra recursos del Estado redistribuyéndolos encre la población, sino como un "padrino" más o menos dispendioso que concede ofrendas a parriculares quienes -por esta vía- son rescatados de entre la anónima muchedumbre y discinguidos como sujetos, como felices beneficiarios de la dádiva.

Así, a los ojos de las expectanies mayorlas, el lider populisia no pareciera gobernar porque fuese ésa la función -y obligación-pública para la cual fue electo, sino que pareciese hacerlo por decision o sacrificio propio. De alli que el vínculó que une a los gobernados con el lider 
populista no sea sólo el del inrerés crematístico sino otro de origen simbólico, menos transparente, asociado a la gratitud por lo que -se creees un Favor, una deferencia, y no un legírimo derecho.

Lo que desde hace ańos ocupa ini interés es precisamente el modo en el que, gracias a las prácricas discursivas públicas que circulan a través de los medios masivos, se consuma can peculiar conversión simbólica. Dicho de orm modo, me he concenrrado desde hace ya algún riempo en explurar las estraregias reróricas que permiren al líder construir las significaciones sociales imaginarias que lu proveen, tanto a él mismo como a sus prosélicos, de una identidad polícica a la que corresponde una práccica empírica concreca, unos modos de hacer, pensar y sentir concordes con uno de los imperarivos máximos del populismo, a saber, el sometimiento por gratirud.

He señalado que esras prácricas discursivas proveen no sólo a la muchedumbre sino iambién al propio líder de un mudelo de interprecación del hacer polírico colecrivo que él comanda, para enfarizar el hecho de que el discurso populista no es necesariamente sinónimo de demagogia, de lúcida manipulación o calculado abuso por parte del gobernante. Con frecuencia, el líder populista es, en lo fundamenral, un creyenre, un convencido de su misión histórica, un convocado, por la historia misma, a redimir. Ello ha sido así cuando menos en las primeras etapas de los procesos merced a los cuales se han insricuido los regímenes populisras; erapas en las que aún el ejercicio del gobierno no ha pervertido al nuevo grupo gobernance $y$ en las que el propio líder funda su prédica en la fuerza de una honesridad aún no envilecida por el imperarivo de conservar, a roda costa, el poder y sus ya degustados privilegios.

Venezuela ha vivido en el siglo XX dos momenros históricos que, a nuestro juicio, se corresponden en especial con el modelo que hemos venido esbozando: el que se inicia con el rriunfante golpe de escado del 18 de ocrubre de 1945 cuyo líder fundamencal fue Rómulo Betancourr, $y$ el que se inicia con el fallido golpe de escado del 4 de febrero de 1992 cuyo líder es Hugo Chávez Frias, que se concreta con el ascenso al poder de este úlrimo el 6 de diciembre de 1998.

Debe quedar claro. No afirmo que tales procesos sean idénticos. Afirmar ral cosa sería -en el menor de los males- una simplificación hisrórica inadmisible. Lo que sí puede afirmarse es que, en ambos procesos, las práccicas discursivas de sus respecrivos lideres han jugado papel protagónico y que, lo han jugadn, en la medida en que han hecho posible la insriución de un modelo particular de interpreración del ejercicio del poder. En ral modelo de interpretación, los líderes emergentes -Betancourt y Chávez- se presentan como "mesiánicos mecenas" dispensadores de la dádiva y su concradiccorio concrapeso simbólicu el pueblo- como una suerte de heroica masa mendicante. 
En este orden de ideas, el ubjetu de nuestra investigación fue hacer explicitas las afinidades que hermanan ambos discursos $y$, a parrir de ellas, reconsiruir las significaciones sociales que componen el imaginario populista que. desde 1936, viene proveyendo a los venezolanos del modelu de interpreración con base en el cual enrendemos, valoramos y reglamentamos nuestras relaciones sociales con el poder.

\section{Corpus y procedimientos}

¿Cómo procedimos enronces? Parriendo de Bulívar (1993, 1994. 1995, 1997, 1999). Fairclough (1989, 1992. 1995) y Fairclough y Wodak (1997), optamos por concentrarnus en el análisis de los rópicos recurrenres en el discurso de los dos líderes que nus cunvocan: Rómulo Berancourr y Hugu Chávez Frías. Para ello, seleccionamos en ambos casos una muestra represencariva de rexcos con énfasis especial en los discursos, entendiéndose en este caso por "discurso" no lo que hemos venido definiendo hasta ahora sino un tipo específico de texto: aquél concebido para ser pronunciado ance una concurrencia conscituida, bien por oyentes presenciales (audiencias masivas o especializadas como, por ejemplo, industriales, académicos, diplomáticos, etc), bien por audiencias mediológicas.

En el caso de Berancourr, amén de la correspondencia, los arrículos de prensa y los libros publicados por el autor entre los años 1936-1948, se analizaron 75 discursos pronunciados por el fundador de Acción Democrática en el lapso que nos atañe, con énfasis especial en aquellos que fueron retransmitidos por la radio a coda la nación. En el caso de Chávez Frías, se seleccionaron 35 discursos de los pronunciados por el acrual Presidente de la República en el lapso 1999-2000, la mayoría de los cuales fueron cransmiridos ianro a rravés de la radio como de las televisoras en cadena nacional. Esta selección de discursos se complementó con las noricias sobre el primer mandacario aparecidas en $E l$ Nacional. El Universal. y Tal Cual entre enero de I99 y octubre del afo 2000.

Elegidos los textos, se procedió a segmentarlos en nraciones ortográficas (Bolivar, 1994: 139-40) y a agruparlos, intertextualmenre, de acuerdo a los rópicos y subrópicos afines tratados en los distintos discursos por el orador. Para establecer la afınidad tópica, se parrió de la presencia en el rexro de vocablos especíticos, de sus sinónimos y/o sus asociados semáncicos (para el caso del pueblo, por ejemplo, calificaron entre ortos soberano, masas populares, mayorias populares y, en algunas ocasiones dependiendo de las marcas semánticas atribuidas, calificaron asimismo vocablos como venezolanos, ciudadanos, pats o nación). 
Asi agrupados lus rúpicus y subrópicos, se procedió entunces al análisis esrableciendo, primero. cuál era el conjunco de palabras o vócablos a los que recurrentemente apelaba el líder populista en sus discursos. Segundo, cuál era el repertorio de significaciones sociales a los que se asociaba cada uno de esros vocablos. Tercero, qué articulaciones más a menos orgánicas se establecían ranco entre las palabras así reconocidas, como entre los significados arribuidus a cada una de ellas.

Del análisis, emergió entonces lo que pudríamus cunsiderar el núcleo duro del imaginario populisra que comenzó a gestarse, circular e inscituirse en el país a la muerte de Juan Vicente Gómez el 17 de diciembre de 1935, si bien algunas de sus significaciones suciales más poderosas - la fascinación por el mando personalizado e incluso aurocrárico para cirar sólo una-hincan ralces en el siglo XIX y aún antes.

\section{Resultados}

\section{I) La APELACIÓN A LOS AFECTUS}

El primer elemento significarivo que ha puesto en evidencia el análisis, es que el imaginario populisra no es sólo un modelo para conocer y/o comprender el mundo en lo que a la prácrica polírica se refiere, sino que simulránea y yuxrapuestamente es un modelo de creencia y de pasión. La polírica comporra siempre un eje irracional, emorivo, incluso lúdico que el discurso populista exacerba hasta persuadir a los oyenres de que se explican cosas, se analizan números, se exponen causas cuando, en el îndo, se está básicamente apelando a la excitación de los a feccios.

Ilusiremos el punto con el caso de Berancourr. Era a la compasión. casi a la lásrima, a las que convocaba el discurso berancourisca cuando sostenía que:

veíamos a los hijos de lus campesinos rachirenses con el pie descalzo y afronrando con una ruana desirnzada el inclemente frio parameno [...] hemos visro a los niños con el estómago inflado por los parásitos intestinales [...] hemos visto los pescadores del Gollo, viviendo en sus ranchos miserables, y a la genie de Nueva Esparta. sedienta, clamando por agua pocable para la Isla. (B-57)

Lo que impele a la lásrima no es la descripción de lo visio, sino la excesiva adjecivación que carga dramáricamence ese mirar: es el descalzo pie; la ruana destrozada; el inclemenze frlo; los esrómagos inflados; los ranchos mierables; la gente que, sedienta, sólo puede clamar

En este mismo orden, era al resentimiento y no al razonamiento al que conminaba Berancour c cuando argüla que

Se ha dichn por las sociólogos del pesimismn, por los enemigos del pueblú de Venezuela, que ésra es una colecrividad inepra para el ejercicio de la vida civili que 
somos un puebló primitivo que sólu puede ser gubernado con el rebenque del autócrata. (B-35).

Y era a la indignación a la que convocaba Betancourt al preguntarse si:

¿Es que somos colecrivamente una nación de demenes o de serviles crónicus, obligados a estar siempre conducidos por el cayado de unos cuanros tutores...? (B19)

Era de esperarse que los oyenres escallaran en cólera al saber que algunos los cenían por inepios, primicivos. dementes, serviles. Con ral muestra de insultos, no se estaba invitando al audicorio a refutar inexacros argumentos; se le estaba "calentando la oreja", se le estaba conminando a ripostar con un rotundo "no" a la agraviante interrogación rerórica que lo describía como menos que los menos.

Escraregias similares hemos hallado en el discurso del actual Presidenre de la República. Obsérvese cómo. por ejemplo, Chávez va inflamando el ánimo de sus seguidores hasta predisponerlos hacia el revanchismo y la inclemencia frente al adversario. En una primera inviración, el Presidente convoca a sus prosélicos a dar una "paliza" a sus enemigos:

[...] Asi como les dimos soberanas palizas el 6 de diciembre, el 25 de abril, el 25 de julio y el 15 de diciembre, igualiro les vamos a dar otra soberasa paliza el próximo 28 de mayo [...] De esa paliza no los salva nadie. Se salvarán de un pasmo, pero no de la soberana paliza que les vamos a dar (...] (CH-7)

Esrá dicho. Los fanácicos saben que el líder no quiere conceder. Y, el líder, embriagado por su propio desafío, remacha el uliimácum para que nadie abrigue dudas:

Usiedes saben que la figura del knock our en beisbol la inventaron para evitar la humillación, es decir, un equipo que no renga ya pircher ni jonrón y palo y palo, y renga diez carreras por encima... entonces, de repente el umpire dice: Knock our... Pero aqul no vale knock out. Yo lo lamento mucho pero aqul no vale knock our, vamos a ganar 45 a cero. (CH-7)

Es imposible roda vuelca arrás. La mulrirud saliva, enardecida, ante la oferra de la presa. Incluso para Chávez, que resience su propia saña. es imposible todo mariz: intenta recoger velas, pero ha sembrado vientos y ha cosechado tempescades:

También, como nosouros snmos caballeros. a veces unn deja que el adversario haga una carrera para que salve la honilla, ¿vamos a dejarlos que hagan una carrera? EJ pueblo grita: ¡Noooo!

Usıedes son más implacables que yo: 45 a una ¿ní se sônlorman con eso? Vamos a ver, levanten la mano los que quieran ganar 45 a 0 .

Bueno, ganaremos 45 a 0 . No vale knock out.. (CH-7) 
Chávez consrara que no hay disposicion a la clemencia y se suma de nuevo a los excesos de lo que ya no es una mulricud sino una rurba. Enardecido por su propia voz, el Comandance llama finalmente al exterminio:

Que se unan codos, yo lós inviro a que de una vez se quiren la careca y que se unan rudos en una sula candidarcara purque les vamos a pasar por arriba iambién. los vamos a artollar rambién. Va a quedar el pulvito (Ера, epa, no me malinrerpreren) $\mathrm{Va}$ a quedar... ni el rastro va a quedar. ( $\mathrm{CH}-7$ )

Lo hemos dicho. Parecen argumentos mas son, en realidad, requisitorias a los afecros. El discurso populisia no puede no conmover. Mas aún. Es de hecho irrelevance a qué afecto se convoca. Lo que es imperarivo es regresar, una y otra vez, al regiscro de la pasión. Obsérvese cómo. por eiemplo. Chávez Frías concluye el periplo de furias que hemos descriro anies con esia convocatoria a la fracernidad que, incluso, podemos admirir como genuina, tan genuina en su concradicción como la previa convocacoria al ensañamiento:

una persona que quiera ser líder y ande destilando veneno y ande descilando rencor y ande descilando odio $y$ ande descilando envidias ; qué puede estar aspirando a dar ejemplo a un pueblo que lo que quiere es amor del bueno? Amor verdadero. Amor. amor maravilloso. Eso es lo que nosotros tenemos por dencro. Eso es lo que nos impulsa, esta pasión: E] amor, el amor. el amor ¡Que viva el pueblo que ama! [...] (CH-7)

Ello es así porque la emoción, los afectos, son el arajo más expedito para alcanzar el mundo incerior de la persona que escucha. Y, en última insrancia, la relación del lider populista con sus seguidores -es decir, con "su" pueblo-, debe necesariamente personalizarse y devenir individual para, según hemos sefialado, abrir caminos a la construcción imaginaria de la "dádiva" $y$, de alli, al somerimiento por gracitud.

\section{2) El DLALOGO DIRECTO}

La apelación a los afecros es, enconces. una de las estracegias recóricas que concribuye a la transmuración de un vínculo que es eminentemente colecrivo e impersonal -el del gobierno-, en una suerte de nexo ínrimo que se transa entre el líder populista y cada gobernado.

A ral meramorfosis concribuyen otras estrategias. La primera, desmicificar el propio discurso, desestruccurarlo como monólogo, imprimirle el carácrer de una conversación, de un diálogo entre el líder y cada uno de lus oyentes que, asl, se ven envuelros en una armósfera incimisca. casi cómplice. Lo hizo Berancourr en el 45:

[...] Es oportuno este momento para que... el Presidenre se dirija a la Nación. Lo haré en el sencillo lenguaje de quien conversa con el pueblo, en lenguaje del pueblo. 
Seré una vez más consecuence can el nuevo esrilu de gubernar i... el cual hall:ı uria de sus más acusadas expresinnes en esta llana franqueza y en esie acenou de sinceridad cũn que lus mandacarios se dirigen a sus corıciudadanos (B-52-A).

\section{Y lo hace Chávez en 1999 :}

no precendo que [esio] sea un discurso grandilucuenre, ussedes ya me onnourn hermanos, mi mensaje no va dirigido a eruditos [...] estas, mis palabras llanas y sencillas, van dirigidas a usted amigu. a usied amiga, a usted amiguica y amiguico. quiero llegarle con mi palabra al humbre del común, a la mujer del cumún. al juven del común, a usiedes jóvenes venezolanos. (CH-9)

La segunda escracegia asoma ya en esce úlrimo rexro y consiste en incerpelar al audirorio direciamente, desmembrándolo, refiriéndose a cada una de las personas que excuchan; conminándolas a que evoquen sus casas, sus vidas, su indivisible experiencia personal; numbrándulas incluso como entidades genéricas - usted amiga, usted amigo- que, por efecto del acto mismo de ser nombradas, abandonan el anonimaro que les imprime el sustancivo común para devenir sujetos. individuos específicos que, así, se sienten mirados, elegidos por el lider de entre la muchedumbre. Hugo Chávez Frías es particularmente diestro en esce caso:

Ahora, analicen eso, háganlo. Esca noche lleguen a su casa y pónganse a hacer un cuadro y díganle a sus hijos: mira, ven acá, vamos a ver el movimienco de escos acrnes poliricos en los úlrimos ocho años. $Y$ eso va a quedar en un gráfico y lo van a ver facilico. hasıa los niños. Esıa niña que esıá aqul lo va a entender faciliro, everdad mi amor? (CH-7)

Puede recurrirse también a la incerpelación, desde el discurso. de personas concretas, de "carne y hueso", conocidas por los oyentes y que, en ese instance, forman parte del mismo audicorio y se confunden con la mulricud. En la medida en que el líder populista reconoce a sus amigos en la masa, se curea con ellos, les hace bromas, en esa misma medida le transfiere a la informe multicud el atributo de ser toda ella parce de "su" tribu, de "su" tropa, de "su" juerga. El actual Presidente de la República califica orra vez como aven rajado en escas lides:

[Vean] quién va en línea recra y quién anda zigzagueando, asl, que no encuentra en qué palo ahorcarse. ¿Ah Darío? Hay gente que no encuentra en qué palo ahorcarse. Darío Vivas. Hola vale ¡cómo está el colesterol? 220. Hola Silvia, ¡cómo esrás?. Puros revolucionarios, cará. Freddy Bernal, Silvia Flores, Dario Vivas, Rangel, todos, todos, La Gocha. Y Marisabel. Bueno, a mi me sale lo mío también. Marisabel quiere tener otro muchacho. Bueno, sí, porque Rosa Inés se la pasa en la escuela. Ya escá en la escuela Rosa Inés. (CH-7)

Por úlrimo, puede recurrirse a la estraregia recórica más explícira, merced a la cual. el líder habla dicectamente desde la primera persona y, 
desde el Yo, individualiza el mando, singulariza el compromiso. persona. liza en su única acción y decisión el acro de gobernar a lus orros.

No hallamos esca escraregia en el discurso berancourista del período en esiudio puesio que el fundador de Acción Democrácica se sabía parte de un gobierno colegiado en el que algunos de los orros convites eran milicares $y$, cumu milicares, exigían proragunizar. De allí que Berancuure apelara siempre a la primera persona del plural para referirse a lus acros de gobierno en un esfuerzo por no contravenir a sus suscepribles colegas de Junia.

Chávez, por el concrario, apela en forma recurrente a este arrificio reróricu. En ucasiones. para mostrarse como servidor bien de sus ideales. bien de sus proséliros. En uno y urro caso, el punto es que el oficio de gobierno se vuelve una cruzada personal, voluncariosa, un compromiso íncimo que ofrece incluso en prenda la propia exiscencia:

Para que el pueblo venezolano recupere de verdad su nivel de vida, en ese esfuerzo desde hoy yo comprometo toda mi voluntad... Mi vida, comprometida está con este esfuerzo. Mi vida, en lo adelante, dedicada escará, como Presidente de Venczuela, como uno más de la bacalla, como primer soldado de esra baralla, dedicada de lleno. rodos los días y codas las noches, en la rarea hermosa que usedes me han asignado (CH-3)

En orras ocasiones. el recurso a la primera persona del singular busca compromerer los sentimiencos del lider populista con las penurias de la nación. El resultado es el mismo que en el caso previo: la causa colecriva deviene causa propia, mas en esca ocasión la metamorfosis no se consagra vía el compromiso érico, sino que se consuma gracias a la empacía sentimental:

[...] Indices macroeconómicos... Si, aquí tengo algunos, no los voy a leer, los sabemos. los conocemus cn libros. en estudios y ya me suenan fríos a mí, piefiero ir por las calles a ver, a senric, a llorar como uno llora cuando consiguc los ninos limpiando las rumbas de los cemenrerios, porque de eso viven, como vi en Barinas cl 2 de enero cuando fui al cementerio a poncrle una corona a mi abuela Rosa Inćs y salieron unos niños a decirle a Chávez: "Chávez, no hay cumbas para limpiar, tenemos hambre". Son niños de Venezuela y son también nuestros hijos. Yo tengo cinco, allá están, pero no tengo cinco, todos los nin̂os que me consiga a mi paso, aunque sean los hijos de mis más duros adversarios, rambién yo los considern mis hijos porque ellos son inocentes de las pasiones que a nosotros nos impulsan. (CH-2)

El propio lider regisıra en su discurso la consumación del efecto retórico: los niños se dirigen a "Chávez", lo tutean, le imploran solución para sus predicamencos. Es a Chávez, no al "primer mandatario nacional" sino a "Chávez", a quien los nin̄os dirigen sus lamentaciones.

La individualización del mando gracias al recurso del Yo alcanza en el actual Presidente de la Répública niveles que, por momenros, parecieran inclusó reducic el accó de gobierno a uná caprichosa elección personal: 
He podidu dar un chavazo, ran lácil onmo umarme un calé, porque asl buena parle del pueblo aún lo rectama, pero desde hace ciempo hemas rumado la decisión de derrotar a los adversarios, a los destructores de Venezuela, en su propio terreno, en sus propios campos de batalla. (30/1 1/1999)

Al propio líder lo rraiciona su jerga. Abre diciendo lo que le dicta la vanidad: el "chavazo" es un asunto que sólo compere a su real gana. Cae en cuenca de la escupidez que acaba de decir y se corrige: en realidad es el pueblo el que le esrá pidiendo que dé el aurogolpe. Vuelve a caer en cuenta de que lo dicho es una provocación y se retracra del rodo: las decisiones las tomamos "nosotros", en colectivo, y hace ya tiempo que dejamos las armas a las que no pensamos regresar, por mucho que las rurbas nos imploren la vuelia.

Más allá de la man̂́a con que Chávez consigue zurcir el capore que él mismo ha rasgado, imporra poner de relieve el hecho de que el recurso sistemárico a esta gruesa hilada de estraregias recóricas rermina por atribuir una peculiar identidad al líder populista: aquélla que lo aucoriza a fungir de dispensador de la dádiva.

El lider se ha asociado a significaciones imaginarias que lo muestran omniporente, plantado en el gobierno y dueño del poder, mas siempre unido a la muchedumbre: lo justo para que ésta pueda, a un mismo riempo, rutearle como a un padrino y reverenciarle como a un dios. No importa que la figura sea terrena o divina. Importa que pueda apertrechar. Y ambos, padrinos y dioses, están allí precisamente para eso: para proveer.

Es gracias al discurso comunicado, una y mil veces reperido, que esta meramorfosis se produce. Y consce que no sólo al Iider debe impurarse la ricánica rarea de conscrucción de su idencidad polícica. La idenridad del líder populista es el resulado de una praxis social a la que coadyuvan tirios y troyanos al unísono. Pesa lo dicho por el líder mismo -por supuesto- cada vez que se ensalza en su propio discurso. Pero pesa cambién lo dicho por sus adversarios, quienes en el desafuern de barirle sólo contribuyen a focalizar el inrerés en su persona para, involuntariamence, reforzar la impresión de que rodos los caminos conducen a su ingenio. Malévolo, es verdad, pero ingenio a fin de cuenras.

$Y$ pesan los medios masivos que. para refurar o para adherir, fungen de ventrílocuos del líder populista. Sí. He dicho "para refurar o para adherir". No nos engañemos. Los medios nunca "informan" en asepsia, $y$ no intento con esce juicio acusarlos de un pecado capical. Nadie informa en asepsia. No existe cal cosa como la "pura información". Por lo menos no una cosa que sirva para comunicar asuntos entre humanos. La producción social de significaciones, siendo un fenómeno colecrivo, no

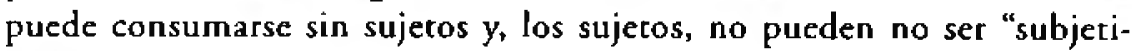
vas". 
De allí que los medios se hallen arrapados en lo que llamaré el "síndrome de las comillas". síndrome que compulsivamente los fuerza a clonar, una y orra vez, lo dicho por el líder. En effecto. reacios a desprenderse de la paz de conciencia que les provoca el miro de la "información objeciva", los jefes de redacción, los reporteros, los redactores, incluso los columnistas cuyo oficio es opinar, se curan en salud y, antes de tronar las hostiles cacerolas $u$ encender los frarernos cohetones, citan texiualmente en rodos los cuerpos, codas las páginas, antes y después de todos los negros y de codas las idas y vueltas a master, citan texcualmente lo dicho por el lider en su apretada agenda-arenga.

Asl, via la reiceración y merced al discurso comunicado -réngase presente a Betancourt: en la lucha politica la consigna es repetir- queda insticuida la identidad y auroridad del líder. Según hemos venido diciendo, este último emerge como una de las significaciones más poderosas que consticuyen el núcleo duro del imaginario que da vida al populismo. La otra, su contrapeso simbólico, omnipresente, es "el pueblo".

\section{3) LA CONSTRUCCION DISCURSIVA DEL I'UEEHIO}

Habría que advertir en primer cérmino que el imaginario populista no se conscruye como una arquitecrura de significaciones más o menos unívocas. más o menos coherentes, articuladas por nexos semánticos más o menos previsibles, sino como una nebulosa ambigua y polinucleada de significaciones, en la que sus varios núcleos -la nación, el parrido, la democracia, el voco-se hallan jerárquicamente referidos a un único sol semántico, polisémico, que es el pueblo. Es al pueblo a quien el populismo ensalza, arerroriza, conmina, convoca, confunde.

Sólo el líder le disputa el protagonismo al pueblo en el imaginario populisra. De hecho, son las prácticas discursivas del primero, masivamenre comunicadas, las que consiruyen una idenridad polírica para el segundo.

Entiéndasenos. El puebla vive y actúa independientemente de que el líder a los medios lo nombren. Pero es el discurso del lider y de los orros actores politicos masivamente comunicado, el que elabora y eventual. mente insrituye un modelo de incerprecación para ese hacer; una manera de valorar ese hacer; una idenridad polírica que se arribuye al sujeco histórico de ese hacer quien, así, queda imaginariamente instiruido como "el pueblo".

Uno de los aspectos relevantes puesros en evidencia por este estudio, es que la idencidad asl construida de "el soherano" resul ra ser una madeja de significados no siempre bien hilada que, por lo mismo. deviene en esencia contradiconria. Efecrivamente, el discurso populista arribuye al pueblu un cünjunco cal de significaciones dispares que, a la postre, ésce 
rermind ora amenazado, ora vicrimizado, y se comporta, ora como cíclope, ora como eunuco; y pasa a la hiscoria ura como liberador, ora como liberado. Lo ĺnico que el pueblu será siempre e inequívocamence. merced a la idencidad que el discurso populista le atribuye, es el Teliz desrinatario de la dádiva. Antes de encrar a ver los ejemplos en cada caso. resultan indispensables algunas precisiones.

Recuérdese que, en úlrimo rermino, unu de lus fenómenus singulares que da piso social al modelo populista es el somerimienro pur la gracicud que los gobernados profesan hacia el líder. Recuérdese asimismo que esra grailud halla su asienco no súlo en la provision de bienes y servicios mareriales sino en el conjunco de significaciones que acompañan esta provisión y que insciruyen imaginariamente la figura de "la dádiva".

Así. la dádiva es mucho más que "las cusas" que el líder parece conceder a cada gobernado en parricular, vía los diversos programas sociales que linancian los recursos de la nación y que se concrean en el ororgamienco a parciculares de algunós bienes fvivienda, alimenous. medicinas), o algunos servicios (acención hospicalaria, telefonía, luz eléctrica). Más allá de "la cosa" cedida, está "la deferencia" prestada; está el "ser tomado en cuenta"; está el ser mirado, amado, protegido, vengado, devuelto a una vida que se ofrece como mejor.

Extrana e incomprensible paradoja, a los ojos de los gubernados, en lugar de deshonrarles, la dádiva les dignifica porque los "saca del montón", porque los convierte en "mimados del poder", porque los transmuta de "anónimos ignorados" en celebridades. Por consiguiente, es en la deferencia donde se ancla, inamovible, la gratitud

Y no es ésta una conclusión ingenua. Conocemos el mensurable peso de lo material. Pero llamamos la arención sobre el inmensurable peso de lo inmarerial. Y ello, porque nos hemos convencido de que sólo desde esta lecrura puede comprenderse la acorazada adhesion que los gobernados brindan a su lider en los regímenes populisıas, y que la hace resiscence incluso a la traición del propio idolo.

De alli que nuestro análisis apunce a detectar las finas estracegias reróricas que permiren al lider hipotecar el agradecimienco de la muchedumbre. La pregunca que oriencó nuestra pesquisa fue, en este orden. ¿qué es lo que el pueblo agradece a su héroe? A concinuación, presencamus nuesiros resulcados.

\section{1) El pueblo AGRADECIDO}

En primer rérmino, el pueblo agradece ser procegido por el líder. Para que tal cosa ocurra, es indispensable que "el soberano" se crea en peligro, bajo amenaza, en riesgo de que algo o alguien le infrinja algún terrible mal. En este orden, la estrategia retórica que permite al discurso populista 
persuadir al pueblo de que se encuentra amenazado, es la construcción discursiva de lo que he definido como "las fuerzas".

Las fuerzas son uno de los más eficientes artificios retóricos de los que dispone el líder para amedrentar a "el soberano". Las fuerzas son entidades ocultas, sin rostro, sin nombre, pero con un alro porencial de desirucción que, agazapadas, mantienen bajo zozobra al pueblo y sus dirigentes.

En palabras de Betancourt, las fuerzas son los "simuladores de preocupación por Venezuela". (B-20); los "enemigos arteros de la Revolación que han prerendido urilizar la arrimaña de dividirnos y de crear una pugna entre los civiles y los militares". (B-21); los "activos organizadores de la guerra civil, fichas movidas en el interior por un rencoroso personaje que rumia sus imposibles ansias de rerorno al poder" (B-52-A).

Las fuerzas son asimismo las que impelen a Chávez a "alertar al país, porque hay gente jugando aquí al caos" ( $\mathrm{CH}-36)$. Son "esos oligarcas mil millonarios que no quieren para nada nuestra revelución pacífica y democrárica" ( $\mathrm{CH}-38)$. Son esos que "andan buscando cualquier cosita que ocurra para ucilizar a los medios de comunicación [...] para arropellar, para mentir descaradamence, para manchar repuraciones, para manchar un pais, para inventar cosas y ponerlas a rodar por radio y por relevisión de manera irresponsable" (CH-39).

Así, las fuerzas brindan al imaginario populista magros servicios. Visro que se comportan como una suerte de caparazón vacio que no refiere a ninguna persona o grupo en concreco, las fuerzas autorizan a que cada quien las rellene con su "canalla" particular, de modo que unos y orros se hermanan en el rencor que creen idéncico y que por lo tanco comparten.

Así, amedrentar al pueblo con las fuerzas es el primer paso para contiscarle su gracitud, roda vez que estas úlrimas permiren al líder populisra proponerse como imbarible redentor del soberano, como paladín de los desvalidos, como infialible conjuro anıe todas las conjuras.

Gracias a las fuerzas, Rómulo Betancourt pudo asegurar en 1946 que:

Fallan los empiesarios de la carástrofe y los augures de caraclismus en sus profeclas o en sus gestiones ancipacrióticas. $Y$, los despechados por la derrota deliniriva que sufricron en la hora de la peripecia armada, ven cómo en el terreno político y adminisrrarivo se consolida In que la sangre generosa de los milicares y civiles caidos [...] conquisró para todos los venezolanos. (B-25).

Y. rambién gracias a las fuerzas, pudo Hugo Chảvez Frías inmolarse y decir que:

a mi podrán hacerme lo que quieran hacerme. A $\mathrm{ml}$ podrán traicionarme los que quieran rraicionarme, a mí podrán apunalearme por la espalda los que quieran apunalesume por la espalda, a ml podrán picarme en pedazos los que quieran 
picarme en pedazos, pero al pueblo venezolano nadie lo va a apufialear por la espalda. Al pueblo de Bolivar nadic lo va a picar en pedazos. Incluso, antes, antes, mil veces, un millón de veces, cien millones de veces, cien millones de veces, hermanos, yo prefiero que ane apubalen eśla espalda cien millunes de veces anaes que vayan a apuñalearle la espalda al pueblo venezolano [...] Y si mi vida tengo que ponerla una y quinienras mil veces para asegurar el camino de la revulución y para evirar que manipulen al pueblo venezolanó, aquí esrá mi vida a la oiden de quien la quiera (CH-7)

Es casi obligarorio que el suberano guarde conmovida graricud pur quienes con ranra hidalguía le amparan del peligro y le brindan ecerna próccción. Comienza de esia forma a contraerse una deuda que, a la postre, devendrá insaldable para la mulricud.

La segunda estrategia recórica que permite al lider enajenar el agrade. cimiento de sus seguidores es aquélla que los postula como "vícrimas" penitentes. En efecro, en el imaginario que alimenta al discursu populisia el pueblo sobrevive no sólo amenazado por las fuerzas sino cambién "vicrimizado", amén de por estas úlrimas y sus secuaces, por la inhumanidad pasmosa de "el gobierno ancerior".

Esca es, sin duda, una de las significaciones más potences del imaginario que da vida al populismo. "El gobierno anrerior" hace de comodín en el mazo de las calamidades que asolan al desventurado pueblo: rodas, absolucamente codas las desgracias se deben a sus desmanes. Para el discurso populista, resulta en el fondo irrelevante quién es en rérminos fácricos "el gobierno ancerior"; el caso es que debe responsabilizársele por la carerva de plagas que azoran al malquerido pueblo.

Responsabilizársele, porque "esas gentes fueron no sólo polícicos despreocupados, sino administradores manirrocos e irresponsables" (B23). Porque "mientras a su parrido y a sus hombres encregaban rodos los recursos y todos los resortes del poder, al pueblo lo mancenían desdeñosamente al margen"(B-13). Porque "una gran cantidad de haciendas pasaron a manos de los aprovechadores sin excrúpulos. y el pueblo se quedó sin rierras" (B-36). Por todas éstas y muchas otras desventuras debe responsabilizarse al "régimen imbuido de orgullo demoníaco y resuelro a mancener a rodo crance una situación que le permila a sus más destacados personeros enriquecerse ilíciramente y uralicar con el parrimonio colecrivo" (B-20).

Es Berancourt quien de este modo esrigmatiza al "gobierno anterior", pero ha podido ser Hugo Chávez Frías. Chávez al afirmar que "los gobiernos que pasaron por aquí l... se acoscumbraron durance muchos ahos a enriquecerse del Tesoro Nacional, a hacet grandes negocios con el tesoro del pucblo, y al pucblo le daban las migajas, la miseria" (CHA-Iy). O Chávez al sostener que "ellos robaron, hicieron negocios... era una macolla negra, nefasta, que destrozó al país'. (CH-40). 
Entiéndase el punto que se intenta ilustrar. El discurso populista se diferencia de úros legírimos discursos de denuncia, porque nu carga el peso semántico -no topicaliza, podría decirse- en las acciones denunciadas o en los viccimarios, sino que se concentra en las vícrimas de esas acciunes y esos verdugus. Ya lo hemos dicho. Son las vícrimas las que inreresan porque son ellas las porenciales oferences de la graricud.

Así, como en el caso de las fuerzas, "el gobierno anterior" no sólo sirve al líder populista para orienrar los udius de las vicrimadas muchedumbres sino que, complementariamente, le permite reperir como salvador que llega a vengar las afrentas $y$ reparar las taras, a "arrancarle a los hombres y mujeres venezolanas esa venda de ignorancia que en los ojos le pusieron los gobiernos interesados en que el pueblo no supiera" (B-36).

Ante tal ofrecimiento, es imposible para "el soberano" no agradecer. Está en deuda con quien se ha apiadado de sus llagas. Está en deuda cun quien ha resarcidu sus honores. Le debe y mucho-al líder que se ha atrevido a castigar a quienes le vienen castigando desde todas las épocas.

\section{2) EI PUEALO CICLÓPEO}

Junto a las marcas semánticas que discinguen a los "enemigos" y al tasado del imporre que, en graticud, el soberano deberá pagar como abono por su prorección, el discurso populista inscicuye rambién los roles que corresponderá cumplir al pueblo ance el relón de fondo de la hiscoria.

Asl, sin olvidar que el uso de su garganta debe ganarle el agradecimiento de las muchedumbres, el líder confiere al pueblo una ambivalente identidad política que lo coloca en el compromecido rol de oscilar entre el estrellaco de la historia -heroico y desprendido- y el pragmatismo de la dádiva que, despojada de sus valores simbólicos, muestra a un soberano egoísta y genuflexo ance la mano que lo ceba.

En el primer caso, el anzuelo hinca en la golpeada auroescima popular que, por efecon del discurso, se ve elevada desde las catacumbas hasra el protagonismo hisrórico. Merced a la jerga populisca, "el soberano" se corona comn parrero de rodos los desrinos. como invencible sujeto que sujera los riempos a su espuela, dominándolos para escribir la hiscoria a sus antojos.

Imporra poco si, en realidad, el pueblo esruvn y ln que hizo esrando. El discurso populista no es jusro sino zalamero cuando exalta las hazańas de la multitud. Y, como todo mortal, el soberano agradece el halago $y$ concede sus gracias en prenda por él.

Así, la primera loa que el discurso populista suele conferir a los ansiosos oldos de la muchedumbre es la que lo encumbra decrerándolo indispensable cuando se trata de acometer heroicas hazañas. Por esta vía el pueblo se escucha nombrado como la cabeza y no como la escoria, el bulto. el lastre de la rebelión. Dírá Berancourt: 
es una verdad indiscrucible que ningún movimienıo hisı́rico ha podido realizarse a espaldas del pueblo [...] porque sólo el aliento vigoroso de las multitudes, la dinámica poderosa de las masas, puede imprimirle rumbos y darle contenidos a cualquier movimiento de transformación (B-2).

La segunda, suele celebrar el proragonismo del pueblo en las campañas que han liberado a la República de sus opresores y que siempre son dos: la de la independencia que lideró Simón Bolivar en el siglo XIX, y la que ha conducido al líder populista hasca el poder. En Becancourr, será Bolivar y la "gloriosa revolución de octubre":

La respuesta del pueblo soberano, la réplica airada de Venecuela a quienes persiscian en considerarla feudo suyo, fue la eliminación definiciva de ese régimen de la vida política de la Nación [...] En consecuencia, la Junta Revolucionaria de Gobierno, esı́a dispuesra a proceder con serena, pero inquebrancable y resuelıa energía. contra quienes pretendan propiciar el retomo a las condiciones polícico-administrativas frence a las cuales insurgió la procesta armada de Pueblo y Ejército. Fraternizando en las calles blusa y uniforme comó en los dias escelares de la nacionalidad, cuandu las masas arresanales y agrarisras, improvisadas para el hernísmo. cơnfundian sus chamarras destlecadas con los rojos dolmanes de los rercios regulares de la milicia libertadora. (D-20).

En Hugo Chávez, será Bolívar y la gloriosa "revolución bolivariana":

Sabido es por todos. en esca tierra bolivariana, que en Venezuela desde hace riempo ya entró en marcha, se puso en marcha un proceso revolucionario que lleva en sus entrahas el mismo signo aquel con el cual comenzó la gesta de Independencia por allá en 1810, en esta misma Caracas, en este valle de los indios Caracas. Por eso, compatrioras, he querido, a pesar de lo ajerreado de la agenda del día [...] venir aquí en este dia memorable de la Pacria, para rendirle triburos desde esta cribuna, al verdadero dueño de esce proceso, al verdadero grandisimo héroe de este ciempo, que no es orro que el pueblo noble y heroico de Venezuela. (CH-3)

Aclaremos el punto. El artificio retórico que intentamos poner de relieve es el de la especulación. El discurso populista no otorga legítimo reconocimiento al papel jugado por el soberano en las luchas sociales. sino que especula con la sobrevaloración que hace de él. Es a la vanidad y no a la autoestima del pueblo a quien emplaza el líder con sus lisonjas. Los individuos con alta aucoestima son menos proclives nadie es inmune- a ser manipulados discursivamente que aquellos que, creyéndose inferiores, se sienten redimidos por un halago que no piensan merecer. El populismo rrafica con Ins complejos del soberano. le hace adicto a sus piropos, pechero de su adulación.

Cabría a estas alturas pregun carse qué nos autoriza a concluir en que el discurso populisra no busca en este caso moldear un pueblo digno sino uno jactancioso. En primer término, la redundancia. Son excesivas -aún cuando en la lucha polírica la consigna sea repecir- son excesivas las 
menciones de este rópico halladas en los rexcos de los dos líderes que escudiamos. En esce orden. el análisis crítico del discurso ha llamado la atención sobre el hecho de que el uso recurrente de un mismo vocablo o rópico puede, bien cargarlo, bien vaciarlo de senrido de mudo que devenga asignificante. Y éste es, a nuestro juicio. el caso que nos ocupa. En especial, porque esia redundancia se complementa con el uso sistemárico de la hipérbule, del halago grandilocuente. No se es "bueno" sino "muy bueno", "noble" sino "noblisimo", "valeroso" sino "heroico".

Así, el pueblo es ensalzado por el líder populisra que, sin mesura, le atribuye cualidades y desrinos riránicos. Veamos el ejemplo en el caso de Chávez:

el pueblo inmenso y ecerno de Bolívar ha resucicado de entre los muerros y aqui esiá. levaniándose de nucvo ante el mundo para demostrá de lo que será capaz. El mundo entero se pondrá de pie para reconocer, para ver, para admirar de lu que es capaz el pueblo venezolano. Nosotros lo demostraremos. Estoy seguro que lo demostraremos, por nuestra dignidad, por nuestra historia, por nuestros hijos, por nuestra moral, por nuestra esperanza. (CH-3)

Es Fénix, renacido, alzando en pie a las naciones que deben no sólo verle sino reconocerle, admirarle, casi reverenciarle. Veamos el ejemplo en el caso de Betancourr:

Aquí está congregada una densa represenración del pueblo venczolano: sólo espera la voz de un comando valeroso para entregarse a una gran faena creadora. Es un pueblo que ya quiere hacer historia, que se resiste a continuar embebido en la estárica adoración del pasado [...] no es la absorca contemplación del ayer lo que essá requiriendo un pueblo que está hoy can capacirado como lo escuvo en esa hora estelar de nuestra historia, la de 1810, para él ser el pionero, el puntero, el baqueano de todo un conninente, en el momento de la marcha resuelia hacia el lururo. (B-8)

El soberano esrá presıo no sólo a liberarse a si mismo sino a remolcaı en la cruzada a la América coda. Más que exalcar, Berancouri se esmera en exacerbar el ego del pueblo quien, merced al discurso populista, se empalaga con las mieles de su propia grandeza.

Mas, el principal indicio que nos permite concluir que esta glorificación del pueblo no es jusıa sino adulante. asoma ya en el úlrimo rexıo citado de Betancourt. Si se lee con cuidado se repara en que el pueblo, en realidad, espera la voz de un comando valeroso para poder comenzar a hacer bistoria. Dicho de ocro modo, el pionero, el puntero. el baqueano, no es el pueblo sino "la voz" que lo comanda.

Así comienza a emerger la concradicción en la idencidad que el discurso populisia atribuye al soberano, visto que no hay modo de ser a un mismo ciempo cabeza y cola de un proceso histórico. No hay modo de serlo, pero esio es jusro lo que se desprende del discurso del líder quien. alternarivamente, se declara siervo y vanguardia de la mulrirud. De esra 
Forma, el líder arribuye al pueblo el dóble rol de amo de su espada y de huesıe de su mando, siendo este úlrimo el papel que deviene hegemónico. Becancour formuló el argumento de manera explícita:

somos desde ahora y para siempre un movimiento enraizado en el pueblo, consustanciado con el pueblo. Somos la vanguardia del pucblo venezolano, técnica y políticarnente organizado dentro de un gran movimiento liberador (B-2)

En el caso de Hugo Chảvez la elaboración es menos obvia, visto que el primer mandarario uscila aún encre el discurso de la parricipación y el de la representación popular. Sin embargo, el ejemplo que sigue no deja dudas sobre el modo en que el Cúmandanre se aurodesigna, a un mismo riempo, cunducror y siervo del soberanu:

Ese pueblo necesita cauce. No podemos defraudarlo de nuevo, no podemos desligurar el pruceso. Asumamus con coraje y con valentía la tarea de darle cauce a la revolucjón vesezolana de este tiempo o la revolución nos pasa por encima [...] (CH-2)

Yo a ustedes les pertenezco, pueblo hermono de Venezucla, pürque amor con amor se paga, y yo no tengo más nada que darles que amor, entrega, trabajo, mi vida entera se las regalo. Ustedes la merecen hermanos (CH-3)

Con rodo, incluso en el rol de masa el pueblo es un agente activo; necesicado de mando, sí, pero acrivo a fin de cuentas, con algo que decir en lo que al diseńo de su vida furura se refiere. La verdadera dislocación que vive la idencidad polícica del soberano merced a las estracegias reróricas desplegadas por el discurso populista es aquella que lo transmura de agente en pacience. de luchador en receptor, en sumiso beneficiario de la dádiva

\section{3) El. pueblo genuflexo}

La metamorfosis ocurre cuando el lider ha romado el poder y tiene acceso a los recursos del Estado. Según ya dijimos, puede entonces iniciar programas de ayuda social donde la redistribución se individualiza (el vaso de leche, la solución habiracional, el uniforme escolar, erc) y donde el propio lider entrega personalmence no sólo "la cosa", sino el complejo universo de grarilicaciones a las que hemos venido haciendo referencia $y$ que concurren para constituir, junro a la marerialidad del objeco concedido. la polimorfa figura de la dádiva.

La identidad politica del pueblo se conforma, así, a la del papel de benefactor que el líder se auroatribuye en su discurso. De las múltiples funciones que un dirigence desempeña en la gesción de gobierno, la única que será recurrentemenre destacada por la recórica populista será la de proveedor. El líder provecrá primero y antes que ninguna orra cosa resuros inmateriales que el pueblo habría extraviado merced a los 
desafueros de sus antiguos verdugos. En el caso de Berancourr, por ejemplo, el entonces presidente afirmaría que:

Uno de los compromisos que adquirimos, inmediatamente después de llegar al Gobierno, fue el de devolverle al pueblo su soberanía usurpada. Pero hay orra, estimados amigos, que creemos de mayor valor: hemos devuelto al pucblo de Venezuela su fe, su confianza en si mismo, su orgullo autóccono, su fervor nacionalista y americano. (B-66)

Ya se ha dicho. De acuerdo con el discurso populista, el pueblo tiene contraída con el líder una deuda insaldable que compromete su voro y su incondicionalidad frente a quien le restituye su derecho a ser, a creer y a decidir.

Amén de por estos bienes intangibles, el líder se configura asimismo como máximo proveedor merced a la oferta y eventual aprovisionamiento de los necesitados y codiciados bienes materiales.

Así, "educar, sanear, alimentar y domiciliar mejor y más racionalmente al pueblo" (B-23) será estribillo en la retórica populista, visto que "la obra de reconstruir la nacionalidad, de alimentar y domiciliar mejor al pueblo. de rescatar del atraso y la miseria a la mayoría de los venezolanos" (B-25) será siempre la primera obligación de "los hombres que han recibido de sus pueblos el honor insigne y la dura tarea de dirigirlos" (B-51)

Por lo dicho, el discurso populista insistirá entonces en enumerar los planes, programas $y$ proyectos en los cuales el pueblo juega el rol de alimentado, domiciliado, saneado, vestido. El líder le conminará cada vez menos a hacer cosas y cada vez más a recibirlas hasta que, finalmente, lo convoque sólo a esperar por ellas. La promesa se erigirá, así, en el hábil artificio que mantenga al soberano asido al líder, más allá de que este último honre o incumpla sus ofrecimientos.

De este modo, la retórica populista cierra el ciclo que le permice anular discursivamente al pueblo: comienza ensalzándole visto que suele ser la fuerza que lleva al líder al poder o, en su defecto, que en él lo legicima. Más, una vez alli, las significaciones que marcan semánricamenre al soberano mutan y transmutan de cal modo, que luego de haber sido la horma del paso vencedor de los vencedores, la riada que movía los molinos, la savia del proceso libertador, el pueblo deviene, por efecto del discurso populista, en dismínuido esclavo de la dádiva.

La dádiva se insticuye entonces como la única significación imaginaria asociada al pueblo. Por ella, el soberano encadena su porvenir al del líder que le concede la migaja. Por ella, la muchedumbre se conforma con el efecto narcotizante de la promesa preterida. Por ella, la multitud deviene en quejumbroso séquito que forma filas a las puertas del recinco donde el lider despacha. 
Y será éste el futuro del soberano. más allá de que el lider populista le renga previstos descinos más nobles y dignificantes. Según reza ur inclemente dicho popular, de "buenas intenciones" está empedrado el camino que conduce al intierno y, la investigación que respalda este estudio. sugiere que bajo el populismo no es posible otro destino para ese soberano que no sea el que aqui se ha resumido y que no lo hace grande sino eunuco, castrado - entre otras cosas- por las atrofiantes lenguas de sus líderes que se empecinan en reducirlo al degradado rol de mendicante.

En este sentido, la historia riene aún alguna cosa que ensefiar a la polirica Y no sólo la hiscoria que enaltece. También es propia la mal andada, ésa que condujo a Venezuela hasıa aquí, desde el populismo de Rómulo Berancourr hasıa el neopopulismo de Hugo Chávez Frías, aunque los líderes -o sus herederos políricos- denigren de las similirudes que los hermanan.

Más allá del desagrado que pueda ocasionar a los respeciivos adeptos, es imperativo comprender que Berancourr y Chávez son padre y criatura de un mismo ímaginario y de una misma prácrica polírica. Ellos como caudillos y el pueblo como masa son el producco de décadas de desafueros populistas autoproclamados como revolución. Y, en el proceso, el discurso ha tenido -como ha podido establecerse- rodo que hacer.

1. La lerra y el númeró corresponden a la idenrificación asignada a cada discurso en el registrn global de la invesrigaciún doctoral de la autora de la que este arriculo furma parte. Los discursos del corpus fueron:

B-2: Discurso pronunciado por R. Betancourt el 8/3/1936 en el Teatro Metropolicano.

B-13: Discurso pronunciado por R. Betancourt en el Circo Metropolitano el 21/9/ 1944 .

B-19. Discurso pronunciado por R. Berancoure en el Nuevo Circo de Caracas el 17/ $10 / 1945$.

B-20: Discurso del Sr. R. Betancourt radiado a roda la nación el 30/10/1945.

B-21: Discurso pronunciado por el Sr. R. Berancourr en la I Convención de Mandatarios Regionales, Miraflores 18 y 22 de noviembre de 1945.

B-22: Palabras de R. Betancourt en el agasajo ofrecido al canciller de Guaremala, el $6 / 12 / 1945$.

B-23: Discurso radiado de $\mathrm{R}$. Betancourc al pueblo del Táchira el 14/12/1945.

B-24: Conferencia dictada por R. Berancourt en el Inscituto Pedagógico Nacional el $29 / 12 / 45$. 
B-25: Alucución del l" de enero de 1946 dirigida por R. Berancourt a la ración.

B-35: Discurso pronunciado por R. Berancourc en la plaza Bolivar de Valencia el 3u/ $3 / 1946$.

B-36: Discurso pronunciado por $R$. Betancourt, ante millares de campesinos congregados en el distrito Carlos Arvelo el día 30/3/1946.

B-47: Discurso pronunciado por R. Berancourt en la Plaza Urdaneta de Caracas, el $18 / 10 / 1946$.

B-52-A: Alocución dirigida por R. Becancourt a la Nación en el dia del Obrero el I/ $5 / 1947$.

B-57: discurso pronunciado por $R$ Berancourt en San Crisióbal, el 15/8/1947.

B-71: Discurso pronunciado por R. Betancourt en El Silencio el 18/10/1948.

CH-2: Discurso de coma de posesión de H. Chávez en el Congreso de la República el $2 / 2 / 1999$.

CH-3: Discurso de H. Chávez en el Paseo de Los Próceres, en el acro de masas por la celebración de la tuma de pusesión el 2/2/1999

CH-7: Discurso de H. Chávez en la Plaza Caracas el día en que inscribió su candidarura ance el CNE el 16/3/2000. www.glubovisión.com

CH-9: Alocucjón de H. Chávez con motivo de los 100 primeros dias de gobierno del $13 / 5 / 1999$.

CH-36: Alocución de H. Chávez del 7/7/2000.

CH-37: "Chávez: Sin los cambios, nadie salvaría a Venezuela de una guerra interna" en www.el-nacional.terra.com.ve, 30/11/1999

CH-39: "Chávez insiste en que García Morales es utilizado por la oposición", www.el-nacional.terra.com.ve, 9/7/2000.

CH-40 "Chávez lanza plomo huerte a ex militares", www.eluniversal.com, 06/3/ 2000.

2. En los Cuademos de la Carcel son múltiples las alusiones de Gramsci a la crisis de hegemonia, tal como esta, tomada del Cuademo No. 7, parágrafo 38: " [...] Separación de la sociedad civil y la polisica: se ha planceado un nuevo problema de hegemonía, o sea que la base histórica del Escado se ha rrasladado. Se riene una forma extrema de sociedad polírica: o para luchar contra lo nuevo y conservar lo vacilante fortaleciéndolo coercitivamente, o como expresión de la nucvo para destruir las resistencias que encuentra en su desarrollo".

3. Para un esiudio más derallado de la recórica chavista en lo que a la interpelación de los afectos se refiere, véase mi articulo "Los demonios del Comandante", AKADEMOS, vol. 2. No 2, junio-diciembre de 2000 . De esre artículo romamos el siguienre pasaje que ilustra el caso del Comandante Chávez en lo que a este rópico acañe.

4. De nuevo, para un análisis más amplio sobre "las fuerzas" véase mi articulo cirado.

REFERENCIAS BIBLIOGRAFICAS

Bolfyar. A. (1993). El encuencro de dos mundos a través del discurso. En Acosta, Hécror (comp.), Una mirada bumanistica (pp. 81-113). Caracas: Fondo Editorial de Humanidades, Universidad Central de Venezuela.

Bolvar, A. (1994). Discurso e interacción en el texto escrito. Caracas: Conscjo de Desarrollo Cientifico y Humanístico. Universidad Central de Venezuela.

Bolivar, A. (1995). Una metodología para el análisis interaccional del texto escrito. Boletin de Lingüfrtica No 9, 1-18.

Bolivar, A. (1997). El análisis crítico del discurso: Teoría y compromisos. Episteme $N S, 1-3,23-45$. 
Bolivar, A. (I 995 j. Las merafunciunes de la cláusula en español Lingua Americtma 4, 48-66.

Britro Garcla, L. (I)Y8). La máscata del poder. Caracas: Alfadil Ediciones.

Britto Garc(a, L. (1989). El poder sin la máscara. Caracas: Alfadil Ediciones.

CAstorladis, C. (1988). Los dominios del hombre: Las encrucijadas del laberinto. Barcelona: Gedisa,

Castorladis, C. (1989). La institucion imaginaria de la sociedad. Madrid: Tusquets. Castorladis, C. (1998). El avance de la insignificancia. Buenos Aires: Eudeba.

Gramscl, A. (1975). Cuadernos de lo Carcel (3 volúmenes). México: Ediciones Era FalrClough, N.(1989). Language and power. Lundun: Lungman Gruup.

Fairclough. N. (1992a). Discourse and social change. Cambridge: Polity Press.

FaIRCLOGUH, N. (1995). Critical discourse analysis. London: Longman Group.

FaIRCLOUGH, N. y R. Wodak, (1997), Critical discourse analysis. En Teun Van Dijk, (Ed.), Discourse Srudies 2. A Multidiciplinary Introduction. Discourse as social interaction (pp. 258-284). London, Sage Publications.

Madriz, M. F. (2000). Los demonios del Comandante. Akademos 2 (2), 65-86.

REY, J. C. 1976). Ideología y cultura polftica: el caso del populismo lacinoamericano. Poliseia 5, 123-150.

Rey, J.C. (1980). Problemar sociopolicicos de América Latina. Caracas: Aceneo de Caracas.

MaRla FERNANDA MADRIZ es invesrigadora del Insticuro de Investigaciones de la Comunicación. ININCO, de la Faculrad de Humanidades y Educación de la Universidad Central de Venezuela y profesora agregada de la Escuela de Artes de la misma Universidad. Se ha especializado en el análisis critico del discurso polfrico y del discurso mediácico, así como en el estudio de los usos alcernativos y las nuevas recrologías de la comunicación. Es resisca del doctorado en Hiscoria de la Universidad Central de Venezuela y publica regularmence arciculos en revistas especializadas en las áreas de la comunicación, la culrura y el análisis del discurso. Correo electrónico madrizm@camelot.rect.ucv.ve 